\title{
Les enfants avec retard sont plus exposés à la maltraitance et relèvent davantage des services de la protection de la jeunesse
}

\author{
Jacinthe DiOn ${ }^{1,2}$, Célia MATTE-GAGNÉ ${ }^{2,3}$, \\ Marc TourignY ${ }^{2,4}$ et Laurie GAUdreAULT ${ }^{2}$
}

\author{
RÉSUMÉ
}

Les enfants ayant un retard sont plus à risques d'être victimes de maltraitance et d'être impliqués dans les mandats de la protection de la jeunesse en comparaison avec les autres enfants. Dans ce contexte, la présente étude vise à distinguer les enfants qui présentent un retard de ceux n'en présentant pas sur le plan des caractéristiques individuelles, familiales et liées aux services. Ces deux groupes sont comparés à deux étapes décisionnelles du processus d'évaluation et d'orientation d'un signalement à la protection de la jeunesse, soit lorsque le signalement est retenu, et lorsque la sécurité et le développement de l'enfant sont compromis. L'étude a été réalisée auprès d'un échantillon représentatif de 4474 enfants dont le signalement a été retenu à la protection de la jeunesse, et d'un échantillon représentatif de 2278 enfants dont la sécurité et le développement ont été jugés compromis. Les analyses de régressions logistiques révèlent que plusieurs caractéristiques individuelles, familiales et liées aux services distinguent les deux groupes d'enfants (avec un retard et sans retard) dans les deux échantillons, notamment la présence de négligence, le jeune âge de l'enfant, des parents vivant de l'aide sociale ou qui ne coopèrent pas avec les intervenants de la protection de la jeunesse. Chez les enfants en retard, les intervenants considèrent plus souvent que les faits fondés impliquent que le développement et la sécurité sont compromis. La prise en charge est plus souvent judiciarisée ou volontaire et les intervenants identifient davantage de besoins de services. La discussion porte sur les facteurs pouvant expliquer ces différences.

MOTS CLÉS : PROTECTION DE LA JEUNESSE, RETARD DÉVELOPPEMENTAL, DÉFICIENCE, ENFANTS, MAUVAIS TRAITEMENTS ENVERS LES ENFANTS

${ }^{1}$ Université du Québec à Chicoutimi

${ }^{2}$ Centre de recherche sur les problèmes conjugaux et les agressions sexuelles (CRIPCAS).

Email: grenouille_71@hotmail.com

${ }^{3}$ Université de Montréal. Email: celiamatte@hotmail.com

${ }^{4}$ Université de Sherbrooke. Email : Marc.Tourigny@USherbrooke.ca

Correspondance : Jacinthe Dion, Département des sciences de l'éducation et de psychologie Université du Québec à Chicoutimi 555 boul. de l'Université Chicoutimi, G7H 2 B1. Email: jacinthe_dion@uqac.ca 


\section{ABSTRACT \\ Children with delay: a different clientele in youth protection services}

Children with delay are at higher risk of being abused and being involved in welfare service mandates compared to other children. This study aims to compare individual and family characteristics of children with delay to those without delay on two decisional stages of the evaluation and orientation process of a report in welfare services: when the report is substantiated and when safety and child development are compromised. The study was conducted among a representative sample of 4474 children for whom the events reported have been substantiated in welfare services, and a representative sample of 2278 children whose security and development were considered compromised. Logistic regression analysis revealed that several individual, family and service characteristics differentiate the two groups of children (with and without delay) in both samples, including the presence of neglect, young children, and parents living on social security or not cooperating with social workers. Among children with delay, social workers more often consider that the substantiated cases imply that the development and security of the child are compromised. Youth protection services are more often voluntary or resorting to Youth Court. Furthermore, social workers identify a greater need for services. The discussion focuses on factors that could explain these distinctions.

KEY-WORDS: CHILD PROTECTIVE SERVICES, DEVELOPMENTAL DELAY, DiSABILITY, CHILDREN, CHILD MALTREATMENT 
Selon les résultats d'une vaste étude canadienne, 235315 signalements de cas de maltraitance envers les enfants ont fait l'objet d'une enquête aux services de la protection de l'enfance en 2003 au Canada (Trocmé, Fallon, MacLaurin, Daciuk, Felstiner, Black et al., 2005). Dans près de la moitié des signalements, les mauvais traitements ont été corroborés par un intervenant de la protection de l'enfance. Par la suite, ils doivent prendre des décisions difficiles concernant la sécurité et le développement des enfants, afin d'assurer la protection des enfants. Ils doivent cependant réaliser cette tâche en dépit d'un manque flagrant de ressources, et ce, dans un contexte où le nombre de signalements est en croissance au cours des dernières années (Trocmé et al., 2005). Par exemple, au Canada, le nombre de signalements concernant l'abus ou la négligence soupçonnés à l'égard des enfants a doublé en 5 ans (entre 1998 et 2003) (Trocmé et al., 2005). Qui plus est, le nombre d'enfants impliqués dans les mandats des services de protection de l'enfance ayant des besoins physiques, médicaux ou développementaux complexes s'est accru durant la dernière décennie (Marchenski \& Mudry, 2005). En conséquence, la capacité des centres de la protection de l'enfance à répondre à ces besoins spéciaux tend à s'épuiser (Marchenski \& Mudry, 2005). Pourtant, ces enfants sont plus vulnérables que les autres enfants. Entre autres, ils sont plus dépendants des autres, sont plus à risque que leur maltraitance passe inaperçue, ont plus de difficultés à être crus lorsqu'ils dévoilent un abus et sont plus isolés (Strickler, 2001).

Bien que de nombreuses recherches aient été conduites à l'échelle internationale concernant les enfants signalés et pris en charge par la protection de l'enfance au cours des trois dernières décennies, très peu d'entre elles ont porté sur les enfants qui présentent des besoins spéciaux. Mais qu'entend-on par « besoins spéciaux »? Divers termes sont utilisés dans la littérature pour désigner les enfants ayant des besoins spéciaux, variations qui reflètent une évolution des connaissances et une réaction à la connotation péjorative de certaines notions, telles que le retard mental. Dans les écrits anglophones, les besoins spéciaux sont désignés par le terme disability (handicap). Cette catégorie très large inclut des enfants qui ont des caractéristiques particulières et spécifiques, ce qui peut rendre les comparaisons entre les différentes études complexes (Fuchs, Burnside, Marchenski, \& Mudry, 2007 ; Westcott \& Jones, 1999). Ainsi, ils peuvent présenter un handicap, tel une paralysie cérébrale, ou encore un retard, tel une déficience intellectuelle. Ces handicaps ou ces retards peuvent être de type développemental, intellectuel, sensoriel, physique ou autre (Fuchs et al., 2007).

Puisque ces enfants sont souvent regroupés dans la même catégorie, rares sont les études qui ont porté spécifiquement sur les enfants qui présentent un retard développemental, intellectuel ou langagier ${ }^{1}$. On dit qu'un enfant a un retard

1 Étant donné que les enfants ayant un retard ont souvent été regroupés dans la plus grande catégorie des disability, ou des enfants ayant des besoins spéciaux, les écrits sur ce thème seront également recensés lorsqu'ils concernent un retard intellectuel, développemental ou langagier. 
lorsque son développement global, intellectuel, ou langagier est significativement en dessous des normes pour son âge. Il s'agit d'une lacune importante, d'autant plus que ces enfants sont plus susceptibles d'être victimes de maltraitance en comparaison avec les autres enfants (Sullivan \& Knutson, 1998 ; 2000). Dès lors, il semble primordial de s'intéresser aux décisions prises par les intervenants lorsqu'un signalement impliquant un enfant présentant un retard est effectué à la Direction de la protection de la jeunesse (DPJ) au Québec.

\section{ENFANTS PRÉSENTANT UN RETARD DÉVELOPPEMENTAL, INTELLECTUEL OU LANGAGIER}

Les résultats d'une vaste étude épidémiologique indiquent que les enfants présentant un retard intellectuel ou langagier ont de 3 à 7 fois plus de risque de vivre plusieurs types de maltraitances (Sullivan \& Knutson, 2000). Ces enfants sont également plus souvent pris en charge par les centres de protection de l'enfance (Weaver, 2005). D'ailleurs, au Manitoba, $30 \%$ des enfants pris en charge par la DPJ ont un handicap, et parmi ceux-ci, une proportion significative présente un retard intellectuel, soit $75 \%$ des enfants (Fuchs et al., 2007).

\section{Caractéristiques des enfants}

Les enfants ayant un retard et qui sont victimes de maltraitance ou référés à la protection de l'enfance sont plus souvent de sexe masculin (Fuchs et al., 2007 ; Sobsey, Randall \& Parrila, 1997 ; Sulllivan \& Knutson, 1998 ; 2000). Les résultats sont plutôt équivoques concernant l'âge de ces enfants, soit qu'ils sont plus jeunes (Sullivan \& Knutson, 1998 ; 2000), ou encore plus vieux (Fuchs et al., 2007 ; Hershkowitz, Horowitz, \& Lamb, 2007) que les autres enfants maltraités. L'étude de Reinke (2005) réalisée auprès de 7672 investigations pour maltraitance auprès d'enfants canadiens indique que la proportion d'enfants identifiés comme ayant un retard décroît avec l'âge.

\section{Caractéristiques de la famille}

La surreprésentation des enfants ayant un retard dans la population des enfants signalés à la protection de l'enfance pourrait être le reflet d'une incidence plus élevée de facteurs de risques liés à la maltraitance dans les familles de ces enfants (Fuchs et al., 2007). Ces facteurs de risques incluent notamment la pauvreté, l'abus de substances chez les parents, l'isolement social et le stress (Fudge Schormans \& Brown, 2005 ; Krahn, Thom, Sokoloff, Hylton, \& Steinberg, 2000). Les enfants qui ont un retard sont souvent considérés comme une source de stress pour la famille (Manders \& Stoneman, 2009). Comparativement aux parents d'enfants au développement typique, les parents (ou tuteurs) d'enfants qui présentent un retard sont moins éduqués, souffrent davantage de problèmes de santé mentale ou physique, ont un lourd passé (ex., maltraitance, activités criminelles), bénéficient 
davantage de l'aide sociale, sont plus souvent locataires et vivent plus souvent dans des conditions de vie non sécuritaires (Fudge Schormans \& Brown, 2005).

\section{Type de maltraitance}

Les résultats des études indiquent que les enfants qui présentent un retard sont plus souvent victimes de négligence, en comparaison avec les autres enfants, et ce, bien que la négligence soit le type de maltraitance le plus souvent rapporté chez tous les enfants (Fudge Schormans \& Brown, 2005 ; Sobsey, 1994 ; Sobsey \& Mansell, 1994). Par ailleurs, chez les enfants qui présentent un retard, même si plus de filles que de garçons sont victimes d'agression sexuelle, les garçons forment une minorité plus grande en comparaison avec les enfants au développement typique victimes d'agression sexuelle (Sobsey et al., 1997). Malgré le fait qu'une proportion significative d'enfants soit victime de multiples formes de maltraitance, ce taux est plus élevé chez les enfants qui présentent un retard (Fudge Schormans \& Brown, 2005 ; Sobsey, 1994 ; Sobsey \& Mansell, 1994). Ils sont également plus souvent victimes de plusieurs agresseurs (Fudge Schormans \& Brown, 2005) et sur des périodes plus longues (Fudge Schormans \& Brown, 2005 ; Sullivan \& Knutson, 2000).

\section{DÉCISIONS PRISES ET PRATIQUES DANS LES SERVICES DE PROTECTION DE L'ENFANCE}

Selon des études récentes, les enfants qui présentent un retard ont moins de risques que leur signalement soit soumis à une enquête, que leur abuseur soit poursuivi en justice (Krahn et al., 2000 ; Perona, Bottoms, \& Sorenson, 2006), ou encore, d'être pris en charge par la protection de l'enfance (Cooke \& Standen, 2002). Ces résultats suggèrent qu'ils ne reçoivent peut-être pas le même degré de protection que les autres enfants. D’autres études réalisées sur les pratiques en protection de l'enfance indiquent que les enfants qui présentent un retard sont plus susceptibles de se voir référer à nouveau à la protection de l'enfance (Connell, Bergeron, Katz, Saunders, \& Tebes, 2007 ; Marshall \& English, 1999). Néanmoins, lorsque le signalement est retenu à la protection de l'enfance, les intervenants sociaux sont plus susceptibles de déclarer que les faits sont fondés lorsque l'enfant apparaît vulnérable, par exemple lorsqu'il a un retard développemental (Fuchs et al., 2007 ; Jones, 1993). Par ailleurs, les enfants qui présentent un retard intellectuel ou développemental ont plus de risques d'être retirés de leur famille et restent plus longtemps en placement (Fuchs et al., 2007 ; Jones, 1993 ; Romney, Litrownik, Newton, \& Lau, 2006).

En raison de la complexité de leurs besoins physiques, cognitifs, comportementaux et sociaux, les intervenants sociaux peuvent avoir plus de difficulté à répondre adéquatement aux besoins des enfants qui ont un retard. Entre autres, ils n'ont pas toujours les connaissances suffisantes et reçoivent rarement une formation supplémentaire pour le faire (Buchele-Ash, Turnbull, \& Mitchell, 1995 ; Cooke \& Standen, 2002 ; Manders \& Stoneman, 2009 ; Orelove, Hollahan 
\& Myles, 2000 ; Shannon \& Agorastou, 2006 ; Westcott \& Jones, 1999). De plus, il n'existerait pas de procédures d'intervention standardisées ou de guides auxquels se référer pour aider les intervenants de la protection de la jeunesse à intervenir auprès de ces enfants (Fuchs et al., 2007 ; Lightfoot, \& LaLiberte, 2006 ; Orelove et al., 2000 ; Westcott \& Jones, 1999), ou à collaborer efficacement avec les organismes qui leur offrent des services (Goldson, 1998). Dans le processus d'évaluation du signalement, le jugement clinique, les préjugés, les attitudes et les croyances des intervenants peuvent influencer les décisions prises (Coicchinelli, 1991 ; Manders \& Stoneman, 2009). Ces enfants présentent donc un réel défi pour les intervenants qui ont à répondre à leurs besoins de services (Krahn et al., 2000 ; Shannon \& Agorastou, 2006).

Dans ce contexte, la présente étude vise à distinguer les enfants qui présentent un retard de ceux n'en présentant pas sur le plan des caractéristiques individuelles, familiales et liées aux services, en comparant ces deux groupes à deux étapes décisionnelles du processus d'évaluation et d'orientation d'un signalement à la protection de la jeunesse soit, lorsque le signalement est retenu, et lorsque la sécurité et le développement de l'enfant sont compromis.

\section{Méthodologie}

\section{Procédure}

Afin de répondre aux objectifs de la présente étude, des analyses secondaires ont été réalisées à partir de la banque de données de l'étude d'incidence québécoise sur les signalements (ÉIQ ; Tourigny, Mayer, Wright, Lavergne, Trocmé, Hélie et al., 2002). Les objectifs de l'ÉIQ étaient d'établir l'incidence de la maltraitance et des troubles de comportements, en plus d'étudier les caractéristiques et les profils des enfants en besoin de protection au Québec. Cette vaste étude a documenté 9790 signalements reçus dans 16 des 18 Centres jeunesse du Québec entre le $1^{\text {er }}$ octobre et le 31 décembre 1998. Les Centres jeunesse des régions administratives du Nunavut et des terres cries de la Baie-James, comprenant respectivement 8000 et 9000 habitants, ont été exclus de l'étude en raison de leur éloignement (difficulté d'accès) et de leur faible population.

Lorsqu'un signalement est reçu par la DPJ, l'étape de « réception et du traitement du signalement »(RTS) permet de déterminer si le signalement est retenu ou non. Par la suite, si le signalement est retenu, l'étape « d'évaluation/orientation » $(\mathrm{E} / \mathrm{O})$ permet une investigation plus approfondie du signalement. L'intervenant vérifie alors les faits signalés, analyse la situation en fonction des conséquences sur l'enfant, des capacités des parents et de leurs conditions de vie. Cette évaluation nécessite des rencontres avec l'enfant, ses parents et toute autre personne jugée significative. L'évaluation permet de statuer dans un premier temps, si les faits sont fondés (c'est-à-dire si l'enfant a effectivement été maltraité) et, dans un deuxième temps, si la sécurité et le développement de l'enfant sont compromis (Comité d'experts sur la révision de la Loi sur la protection de la jeunesse, 2004 ; Jacob \& Laberge, 2001). Par la 
suite, si l'intervenant considère que les faits sont fondés sans que la situation ne compromette la sécurité ou le développement de l'enfant, le dossier est fermé. S’il juge toutefois que les faits sont fondés et que la sécurité et le développement sont compromis, l'intervenant propose des mesures d'orientations visant à faire cesser les mauvais traitements. Parmi ces mesures, on retrouve des mesures de prise en charge volontaires ou judiciarisées (via une ordonnance du Tribunal de la jeunesse), le placement de l'enfant ou une intervention terminale de courte durée.

Dans le cadre de la présente étude, les données utilisées concernent celles recueillies à l'étape É/O. Les signalements retenus et documentés par les intervenants durant la période couverte par l'ÉIQ représentent $86 \%$ (variant de 64 à 99 \% selon le CJ) de tous les signalements enregistrés et retenus dans les centres jeunesse du Québec durant cette même période.

\section{Participants}

Le premier échantillon utilisé pour répondre à l'objectif comprend 4474 enfants dont le signalement a été retenu à la DPJ pour abus, négligence, abandon et troubles de comportements sérieux. Parmi ces enfants, 12,8\% $\%(\mathrm{~N}=613)$ présentent un retard développemental, intellectuel ou langagier. Le second échantillon inclut 2278 enfants dont le signalement a été jugé fondé et la sécurité et le développement compromis.

\section{Variables indépendantes}

Formulaire d'enquête É/O

Les données utilisées dans le cadre de la présente étude proviennent du formulaire d'enquête É/O élaboré aux fins de l'étude ÉIQ en tenant compte : 1. du processus de traitement des signalements propre au système de protection de la jeunesse du Québec ; 2. des attentes exprimées par les intervenants des CJ et les chercheurs impliqués dans l'étude ; 3. d'instruments de mesure provenant d'enquêtes similaires telles l'Ontario Incidence Study (OIS - Trocmé, McPhee, Tam, \& Hay, 1994) et l'étude américaine National Incidence Study (NIS - Sedlak, 1991) ; 4. de la nécessité de recueillir les mêmes informations que dans l'étude canadienne d'incidence (éCI - Trocmé, MacLaurin, Fallon, Daciuk, Billingsley, Tourigny et al., 2001) ; et 5. de l'Inventaire concernant le bien-être de l'enfant en relation avec l'exercice des responsabilités parentales (ICBE - Vézina \& Bradet, 1990). Ce formulaire a été complété par l'intervenant qui a évalué le signalement une fois retenu et a permis de recueillir de l'information sur les caractéristiques : 1. de l'enfant signalé (âge et sexe) ; 2. de la problématique signalée (agression sexuelle, abus physique, abus psychologique, négligence, abandon, troubles de comportements sérieux et présence de plus d'une problématique signalée); 3. des adultes jouant le rôle de parents auprès de l'enfant (degré de coopération avec l'intervenant de la DPJ, évalué à partir de l'ICBE (Vézina \& Bradet, 1990), une échelle en quatre points allant de « adéquat » à « sérieusement inadéquat ", 
présence d'au moins un adulte vivant de l'assistance sociale, origine autochtone et nombre total de problèmes connus ou soupçonnés chez les parents (violence conjugale, alcool ou drogue, criminalité, santé mentale, santé physique, soutien social, retard mental, difficulté économique, séparation ou divorce, autre) ; 4. du milieu de vie de l'enfant (conflits de garde, structure familiale, présence d'un lieu de résidence sécuritaire, type d'occupation du logement, nombre de déménagements dans la dernière année et présence d'un autre signalement dans la même famille) ; 5. des signalements reçus et retenus (signalement antérieur retenu au cours de la dernière année, prise en charge au cours des 5 dernières années, mesures d'urgence, priorité d'intervention accordée au signalement, placement, décisions en réponse à l'évaluation (faits fondés, sécurité et développement compromis), et lorsque la sécurité et le développement de l'enfant sont compromis : la recommandation de l'orientation (volontaire ou judiciarisée) et du placement) ; et enfin 6. des besoins de services sont identifiés (pour l'enfant et ses parents).

\section{Variable dépendante : enfants présentant un retard}

Le retard intellectuel, développemental ou langagier est identifié par l'intervenant chargé du signalement. En se basant sur l'ensemble des éléments recueillis durant l'évaluation, il devait indiquer s'il y avait présence connue ou soupçonnée d'un retard sur le formulaire d'enquête É/O. La présence d'un retard est connue si un médecin ou un professionnel qualifié l'a dévoilé ou a posé un diagnostic à cet effet. Elle est soupçonnée si l'intervenant considère que ses soupçons sont assez importants pour les inclure dans son rapport ou pour en faire part à un collègue qui prendrait le dossier. Les catégories pour les retards connus ou soupçonnés ont été colligées aux fins d'analyses.

\section{Stratégies d'analyses}

Dans un premier temps, des analyses préliminaires bivariées (Chi carrés et Anovas) ont permis de déterminer si les enfants qui ont un retard diffèrent des autres enfants, en regard des caractéristiques de l'enfant, de sa famille et des décisions prises. Deux analyses de régression logistique de type pas à pas (stepwise) ont ensuite été réalisées en conservant comme variables indépendantes seulement celles qui avaient atteint le seuil de signification de 0,05 lors des analyses préliminaires. Ce type d'analyse a été privilégié étant donné la nature exploratoire de cette étude, permettant ainsi d'explorer la contribution des différents facteurs, et ce, sans accorder une importance particulière à certains d'entre eux (Tabachnick \& Fidell, 2007). Les effets d'interaction n'ont pas été évalués, étant donné le caractère exploratoire de cette étude et du grand nombre de variables indépendantes analysées. Les résultats reposent ainsi sur l'hypothèse que le processus analysé est relativement homogène dans la population étudiée. Par ailleurs, les variables catégorielles de plus de deux catégories ont été introduites dans les modèles de régression logistique selon la méthode des 
contrastes dite indicator (indicateur), ce qui permet de choisir la catégorie de référence comme base de comparaison, facilitant ainsi la description des résultats.

\section{RÉSULTATS}

\section{Analyses univariées}

Les résultats des analyses univariées sont présentés dans les tableaux 1 à 3 pour l'échantillon représentatif d'enfants dont le signalement a été retenu à la DPJ. Plusieurs caractéristiques individuelles, familiales et reliées au signalement permettent de distinguer les enfants qui ont un retard des autres enfants. Il est à noter que le même exercice d'analyses univariées a été refait avec l'échantillon d'enfants dont le signalement a été jugé fondé et la sécurité et le développement compromis. Ainsi, les mêmes caractéristiques individuelles et familiales ont fait l'objet des analyses univariées (tableaux 1 et 2). Les résultats obtenus sont similaires, c.-à-d. que les mêmes caractéristiques distinguent les deux groupes d'enfants ${ }^{2}$. Pour ce qui est des caractéristiques des signalements retenus, seules les variables reliées spécifiquement à l'étape décisionnelle du processus d'évaluation et d'orientation d'un signalement des enfants dont la sécurité et le développement sont compromis ont fait l'objet des analyses (c.-à-d.,

Tableau 1.

Caractéristiques des enfants et du type de signalement selon la présence de retard chez les enfants

\begin{tabular}{lcccc}
\hline $\begin{array}{l}\text { Caractéristiques des enfants et } \\
\text { du type de signalement reçu }\end{array}$ & $\mathbf{N}$ & $\begin{array}{c}\text { Enfants avec } \\
\text { retard }\end{array}$ & $\begin{array}{c}\text { Enfants sans } \\
\text { retard }\end{array}$ & $\chi^{2}$ ou F \\
\hline Âge & 4715 & 6,8 ans $(5,2)$ & 9,2 ans $(4,7)$ & $\mathrm{F}(112,55)^{* * *}$ \\
\hline Sexe (garçon) & 4745 & $60,0 \%$ & $51,5 \%$ & $15,45^{* * *}$ \\
\hline Motif de signalement & & & & \\
\hline Agression sexuelle & 4774 & $5,5 \%$ & $6,2 \%$ & 0,46 \\
\hline Abus physique & 4774 & $12,1 \%$ & $12,6 \%$ & 0,15 \\
\hline Abus psychologique & 4774 & $23,2 \%$ & $16,0 \%$ & $19,33^{* * *}$ \\
\hline Négligence & 4774 & $57,9 \%$ & $31,1 \%$ & $169,19^{* * *}$ \\
\hline Abandon & 4774 & $4,4 \%$ & $4,4 \%$ & 0,00 \\
\hline Troubles sérieux du comportement & 4774 & $23,3 \%$ & $34,6 \%$ & $30,67^{* * *}$ \\
\hline Plus d'une problématique signalée & 4774 & $37,0 \%$ & $26,6 \%$ & $29,00^{* * *}$ \\
\hline & & & &
\end{tabular}

2 Ces résultats ne sont pas présentés afin de ne pas allonger l'article indûment, mais sont disponibles au lecteur intéressé par ceux-ci. 


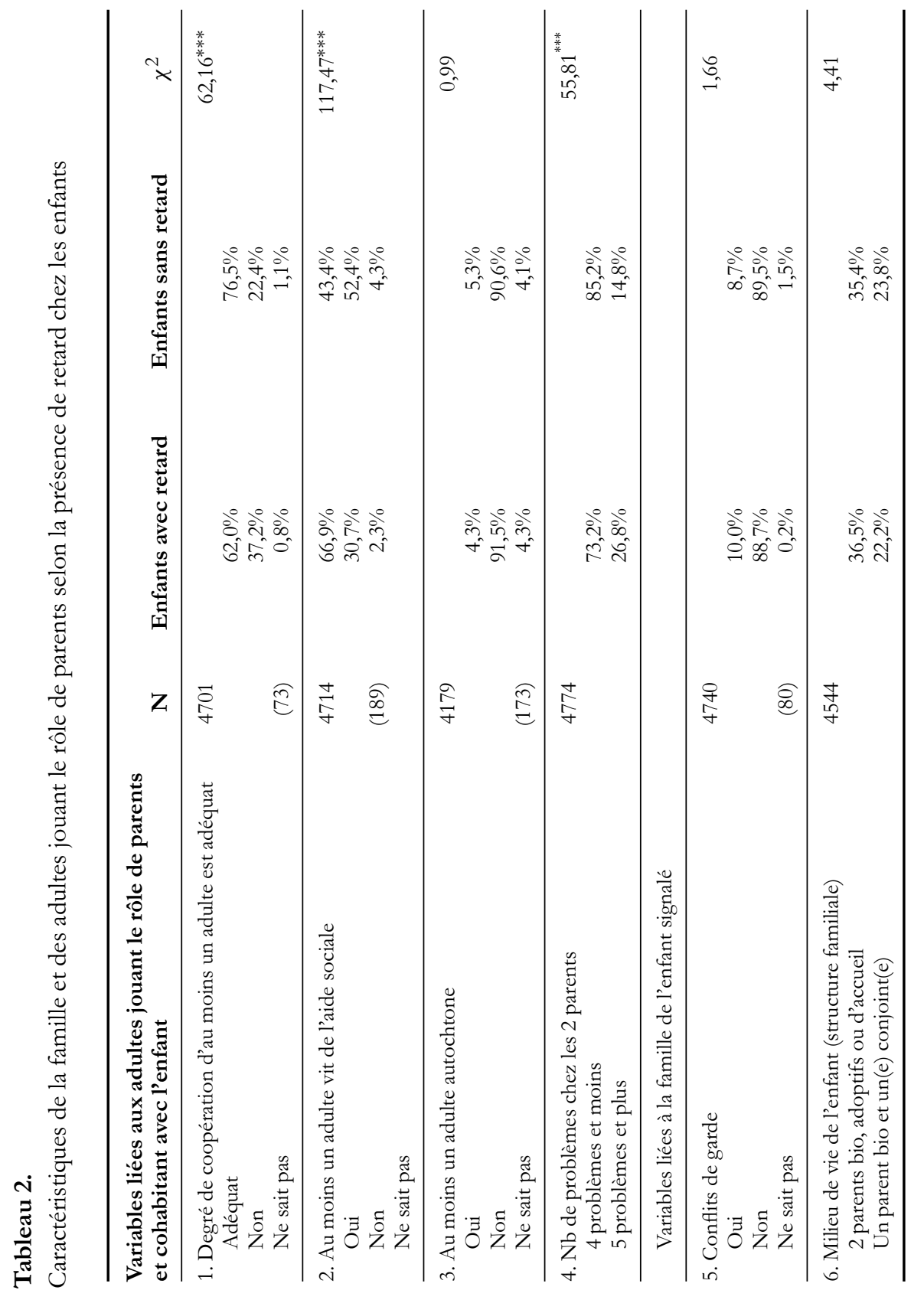




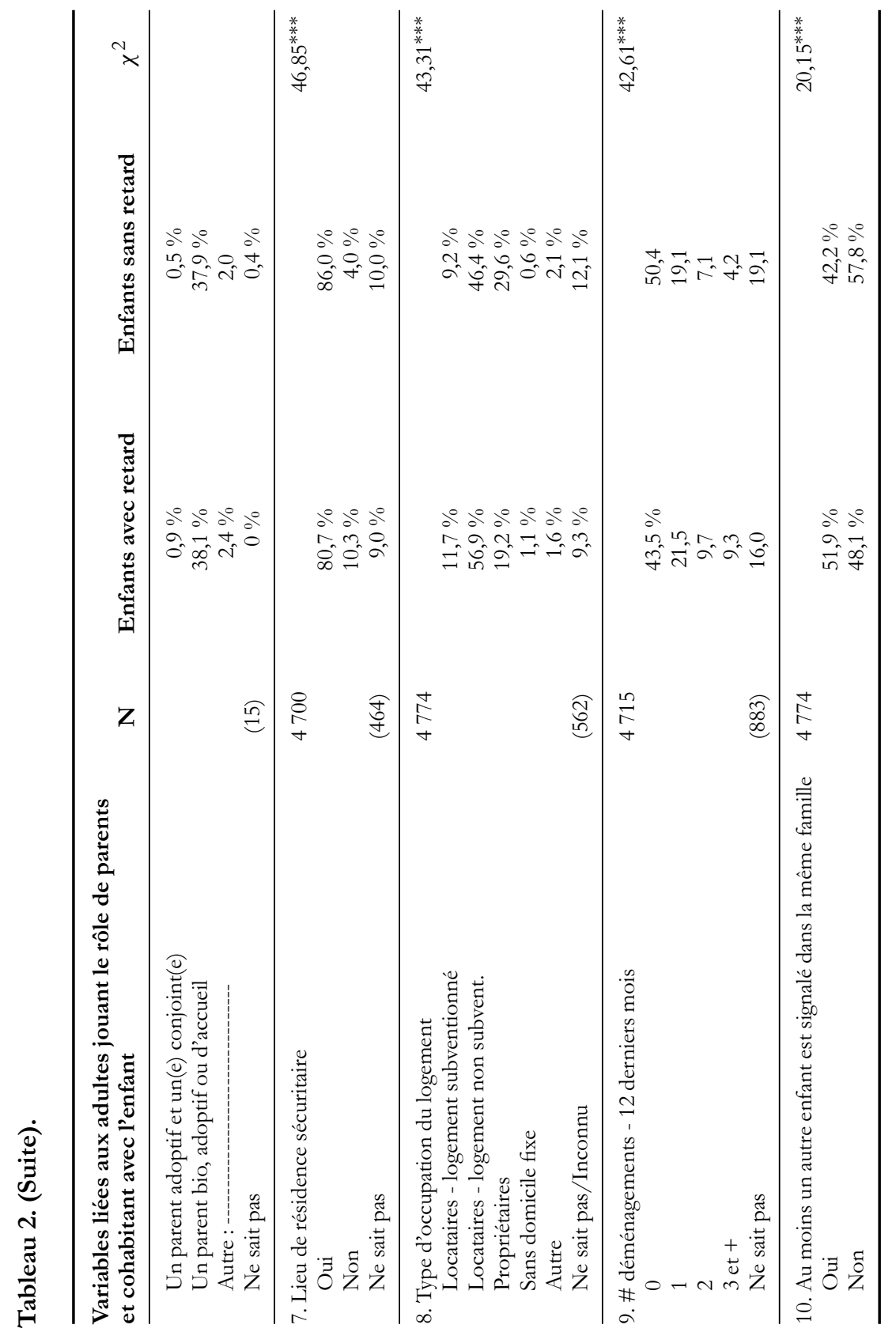




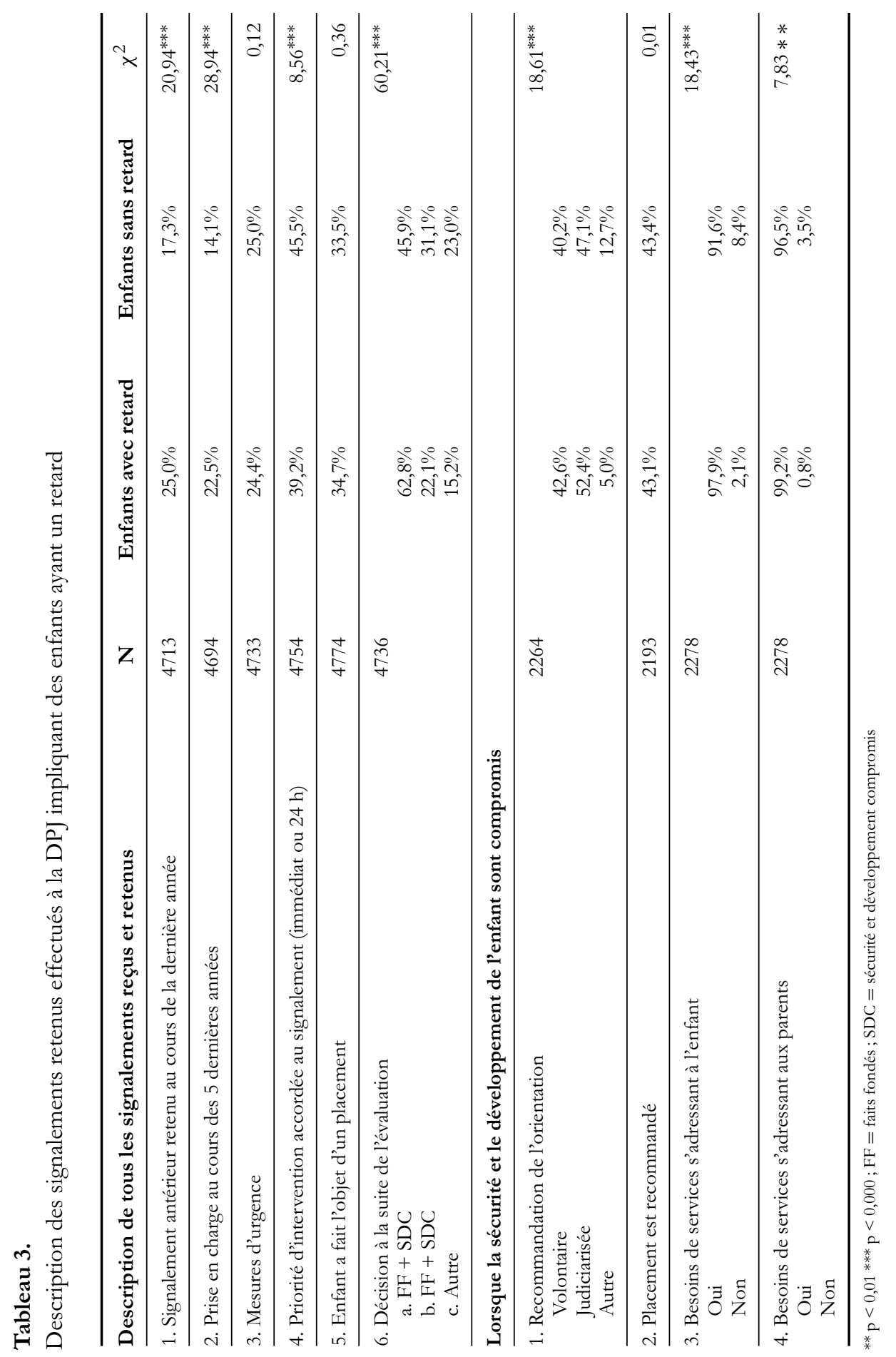


recommandation de l'orientation, placement et besoins de services pour les parents et les enfants - voir tableau 3 pour un aperçu des résultats).

\section{Régressions logistiques}

Suivant les résultats des analyses univariées, deux régressions logistiques ont été réalisées afin de comparer les enfants qui présentent un retard des autres enfants, l'une incluant tous les signalements retenus et l'autre, les signalements fondés et dont la sécurité et le développement de l'enfant sont compromis.

Le tableau 4 présente les résultats de l'analyse de régression logistique qui a permis d'explorer ce qui différencie les enfants qui présentent un retard de ceux qui n'en présentent pas lorsqu'un signalement est retenu par la DPJ. Les 17 variables significatives dans les analyses univariées ont été entrées en mode stepwise dans l'analyse de régression logistique. De celles-ci, huit variables sont

Tableau 4.

Facteurs associés aux enfants qui présentent un retard lors du signalement à la DPJ $(\mathrm{n}=4467)$

\begin{tabular}{|c|c|c|c|c|c|}
\hline \multirow[b]{2}{*}{ Variables } & \multicolumn{5}{|c|}{$95 \%$ IC pour exp. $b$} \\
\hline & B (ET) & Wald & Exp. (b) & Bas & Haut \\
\hline Âge & $-0,08(0,01)$ & $51,60^{* * *}$ & 0,93 & 0,91 & 0,95 \\
\hline Sexe & $-0,33(0,10)$ & $11,99^{* * *}$ & 1,39 & 1,15 & 1,68 \\
\hline $\begin{array}{l}\text { Motif de signalement : } \\
\text { négligence }\end{array}$ & $0,62(0,11)$ & $30,53^{* * *}$ & 1,86 & 1,49 & 2,32 \\
\hline $\begin{array}{l}\text { Un des adultes coopère } \\
\text { adéquatement avec } \\
\text { l'intervenant }\end{array}$ & & $9,22^{*}$ & & & \\
\hline Inadéquat/adéquat & $0,31(0,10)$ & $9,15^{*}$ & 1,37 & 1,12 & 1,68 \\
\hline Inconnu/adéquat & $-0,05(0,56)$ & 0,01 & & & \\
\hline $\begin{array}{l}\text { Un des parents vit de } \\
\text { l'aide sociale }\end{array}$ & & $24,37^{* * *}$ & & & \\
\hline Oui/non & $0,49(0,11)$ & $21,27^{* * *}$ & 1,64 & 1,33 & 2,02 \\
\hline Inconnu/non & $-0,24(0,32)$ & 0,57 & & & \\
\hline $\begin{array}{l}\text { Prise charge antérieure } \\
\text { (5 ans) }\end{array}$ & $0,54(0,12)$ & $19,40^{* * *}$ & 1,71 & 1,35 & 2,17 \\
\hline $\begin{array}{l}\text { Priorité d'intervention } \\
\text { dans les } 24 \mathrm{~h}\end{array}$ & $-0,38(0,10)$ & $15,18^{* * *}$ & 0,68 & 0,57 & 0,83 \\
\hline $\begin{array}{l}\text { Décision rendue suite à } \\
\text { l'évaluation }\end{array}$ & & $13,52^{* *}$ & & & \\
\hline $\mathrm{FF}+\mathrm{SDC} / \mathrm{FF}+\mathrm{SDNC}$ & $0,40(0,12)$ & $11,33^{* *}$ & 1,49 & 1,18 & 1,87 \\
\hline Autre/FF + SDNC & $-0,02(0,16)$ & 0,01 & & & \\
\hline
\end{tabular}

Note. Model $\chi^{2(6)}=328.16, \mathrm{p}<0,000 .{ }^{*} \mathrm{p}<0,01,{ }^{* *} \mathrm{p}<0,001,{ }^{* * *} \mathrm{p}<0,000$. ; FF $=$ faits fondés ; SDC $=$ sécurité et développement compromis ; SDNC $=$ sécurité et développement non compromis. 
ressorties comme étant les plus fortement associées aux enfants qui présentent un retard. Le modèle final, en comparaison avec le modèle incluant seulement la constante, s'est avéré significatif $\chi^{2}(8, \nu=4467)=328,16$; $\mathrm{p}<0,000$. Les 8 variables, dans leur ensemble, distinguent donc les enfants qui présentent un retard des autres enfants. Le résultat du test d'ajustement de Hosmer et Lemeshow $^{3}$ (1980) montre qu'il n’y a pas de différence significative entre les prédictions du modèle et les données observées (Goodness of Fit $=5,39$; $\mathrm{dl}=$ $8 ; \mathrm{p}=0,72)$ ce qui implique que le modèle obtenu reflète bien les données observées. En ordre décroissant d'importance, les facteurs suivants sont associés à une plus grande probabilité que l'enfant présente un retard : 1. le signalement concerne une situation de négligence, 2. il y a eu prise en charge antérieure dans les cinq dernières années, 3. un des parents vie de l'aide sociale, 4. la priorité d'intervention n'est pas accordée dans les 24 heures, 5. les faits sont fondés et la sécurité et le développement sont compromis, 6. l'enfant est un garçon, 7. un des parents ne coopère pas adéquatement, et 8. l'enfant est plus jeune.

La deuxième analyse de régression logistique a été réalisée auprès des enfants dont la sécurité et le développement étaient compromis. Comme mentionné précédemment, nous n'avons pas repris les caractéristiques des services rendus (ex., prise en charge antérieure, intervention immédiate), étant donné qu'elles faisaient l'objet de la première analyse de régression. Ainsi, 16 variables significatives dans les analyses univariées ont été considérées. De celles-ci, le modèle final a retenu 7 variables significatives permettant de différencier les enfants qui présentent un retard de ceux qui n'en présentent pas $\chi^{2}(7, v=2$ $161)=222,34 ; \mathrm{p}<0,000$. La statistique de Hosmer et Lemeshow s'est avérée non significative (Goodness of Fit $=6,13 ; \mathrm{dl}=8 ; \mathrm{p}=0,63$ ), suggérant que le modèle représente bien les données. En ordre décroissant d'importance, les facteurs suivants sont associés à une plus grande probabilité que l'enfant présente un retard, lorsque la sécurité et le développement sont compromis : 1 . des besoins de services sont plus souvent identifiés pour l'enfant, 2. la prise en charge est plus souvent volontaire ou judiciarisée, 3. la situation décrit une situation de négligence, 4. le lieu de résidence n'est pas sécuritaire, 5. un des parents ne coopère pas adéquatement, 6 . un des parents vit de l'aide sociale, et 7 . l'enfant est jeune.

\section{Discussion}

Dans l'ensemble, les résultats de la présente étude révèlent des différences entre les enfants qui présentent un retard et les enfants au développement typique, et ce, à deux étapes distinctes du processus d'évaluation/orientation de la DPJ.

3 La statistique Hosmer-Lemeshow évalue le goodness-of-fit (la qualité de l'ajustement) d'un modèle, où un bon modèle produit un chi-carré non significatif. Cette statistique permet donc d'estimer l'adéquation du modèle aux données observées. 
Tableau 5.

Facteurs associés aux enfants qui présentent un retard lorsque le signalement est fondé et la sécurité et le développement compromis ( $\mathrm{n}=2$ 161)

\begin{tabular}{|c|c|c|c|c|c|}
\hline \multirow[b]{2}{*}{ Variables } & \multicolumn{5}{|c|}{$95 \%$ IC pour exb b } \\
\hline & B (ET) & Wald & $\operatorname{Exp}(b)$ & Bas & Haut \\
\hline Àge & $-0,08(0,01)$ & $38,91 * * *$ & 0,92 & 0,90 & 0,94 \\
\hline Négligence fondée & $0,63(0,14)$ & $19,45^{* * *}$ & 1,88 & 1,42 & 2,5 \\
\hline $\begin{array}{l}\text { Un des adultes coopère } \\
\text { adéquatement avec } \\
\text { l'intervenant }\end{array}$ & & $8,55^{* *}$ & & & \\
\hline Inadéquat/adéquat & $0,38(0,13)$ & $8,48^{* *}$ & 1,47 & 1,13 & 1,90 \\
\hline Inconnu/adéquat & $0,45(0,88)$ & 0,26 & & & \\
\hline $\begin{array}{l}\text { Un des parents vit de } \\
\text { l'aide sociale }\end{array}$ & & $7,17^{*}$ & & & \\
\hline Oui/non & $0,35(0,15)$ & $5,81 *$ & 1,42 & 1,07 & 1,88 \\
\hline Inconnu/non & $-0,38(0,50)$ & 0,56 & & & \\
\hline $\begin{array}{l}\text { Lieu de résidence } \\
\text { sécuritaire }\end{array}$ & & $10,11^{*}$ & & & \\
\hline Oui/non & $-0,38(0,19)$ & $3,81 *$ & 0,69 & 0,47 & 1,00 \\
\hline Ne sait pas/non & $0,20(0,27)$ & 0,52 & & & \\
\hline Prise en charge & & $7,69 *$ & & & \\
\hline Volontaire/autre & $0,71(0,27)$ & $7,18^{*}$ & 2,03 & 1,21 & 3,41 \\
\hline Judiciarisée/autre & $0,53(0,27)$ & $3,99 *$ & 1,70 & 1,01 & 2,85 \\
\hline Besoins services enfants & $2,03(0,40)$ & $25,61 * * *$ & 7,62 & 3,47 & 16,73 \\
\hline
\end{tabular}

Model $\chi^{2(6)}=222,34, \mathrm{p}<0,000 . * \mathrm{p}<0,05,{ }^{* *} \mathrm{p}<0,001,{ }^{* * *} \mathrm{p}<0,000$.

Les résultats montrent que les enfants ayant un retard présentent un profil individuel et familial plus détérioré que les enfants au développement typique. Les facteurs distinguant les deux groupes peuvent être considérés comme des facteurs de risques pour expliquer l'émergence d'un retard, ou à l'inverse, en être une conséquence.

\section{Caractéristiques de l'enfant}

Les résultats de notre étude, chez les enfants dont le signalement a été retenu, sont cohérents avec ceux de plusieurs recherches qui démontrent que la proportion de garçons est plus grande chez les enfants ayant des retards que chez ceux n'en ayant pas (Fuchs et al., 2007 ; Sullivan \& Knutson, 2000 ; Sobsey et al., 1997). Nos résultats indiquent également que les enfants présentant un retard sont plus jeunes que les autres enfants, ceux-ci s'apparentent aux résultats de Sullivan \& Knutson (1998; 2000) et de Reinke (2005), mais sont contraires à ceux de Fuchs (Fush et al. 2007). D'autres études s'avèrent donc nécessaires pour 
mieux comprendre le rôle de l'âge qui pourrait, par exemple, varier en fonction du type de retard.

\section{Type de signalement et caractéristiques familiales}

L'association entre la maltraitance et le retard est bien établie (Fudge Schormans \& Sobsey, 2007). Sobsey (1994) suggère d'ailleurs que de $3 \%$ à $6 \%$ de tous les enfants maltraités vont développer comme séquelle un certain degré de retard développemental permanent. Il ajoute également que les types de retard ou de handicap peuvent être des facteurs de risque de la maltraitance dans $10 \%$ à $25 \%$ des cas. Dans le cadre de la présente étude, il n'a pas été possible d'évaluer la bilatéralité de cette relation, mais plutôt la prévalence des divers types de maltraitances chez les enfants ayant un retard comparativement aux autres enfants. Il en ressort, tel que retrouvé dans plusieurs études, que la négligence est plus fréquente chez les enfants ayant un retard (Fudge Schormans \& Brown, 2005 ; Sobsey, 1994 ; Sobsey \& Mansell, 1994). Un retard ou un arrêt du développement pourrait alors être une conséquence de la négligence des besoins d'attention, de stimulation et d'apprentissage de l'enfant (Fudge Schormans \& Sobsey, 2007 ; Hughes \& Rycus, 1998).

À l'inverse, il est tout aussi plausible que la présence d'un retard chez l'enfant puisse conduire à la négligence. D'ailleurs, plusieurs facteurs peuvent contribuer à la vulnérabilité accrue des enfants ayant un retard vis-à-vis de la maltraitance, notamment leur plus grand isolement, leur dépendance par rapport aux autres, la prévalence élevée des troubles psychopathologiques et leurs habilités de communications plus limitées (Fudge Schormans \& Sobsey, 2007 ; Hvam, 2000 ; Sobsey, 1994 ; Tharinger, Horton, \& Millea, 2000). Les stress familiaux tels le tempérament difficile et le manque d'autonomie de l'enfant, les soins spécialisés requis, la sévérité du retard peuvent également engendrer de grandes difficultés d'adaptation pour la famille et de ce fait, représenter des risques de mauvais traitements (Shannon, 2006).

Par ailleurs, les parents d'enfants ayant un retard peuvent présenter un plus grand nombre de facteurs de risques (p. ex., faible revenu) liés à la maltraitance (Fuchs et al., 2007 ; Fudge Schormans \& Browns, 2005). Plusieurs caractéristiques familiales sont d'ailleurs ressorties à cet effet dans les analyses préliminaires (p. ex., parents ayant plus de problèmes, fratrie ayant fait l'objet d'un signalement), mais trois ressortent particulièrement à la suite d'analyses de régression : une proportion plus grande de parents ne coopère pas adéquatement avec l'intervenant responsable de l'enfant et plus de familles vivent de l'aide sociale ou habitent un lieu de résidence non sécuritaire (c.-à-d., la présence de plusieurs situations dangereuses où l'enfant a subi des blessures ou est à risque d'en subir, p. ex., de la peinture à base de plomb, du gaz s'échappant de la cuisinière). Ne pas coopérer adéquatement fait référence au fait de ne pas s'impliquer dans les interventions, d'être non disponible et de ne pas utiliser les ressources et le soutien offerts. Il est possible que ces caractéristiques soient 
reliées à la présence de psychopathologies ou de troubles développementaux chez les parents. Le nombre total de problèmes identifiés chez les parents n'est pas ressorti comme un facteur qui distingue les deux groupes d'enfants. Cependant, des analyses univariées ont été réalisées a posteriori afin de vérifier si les parents d'enfants ayant un retard avaient des problèmes différents des autres parents. Comparativement aux autres parents, chez les parents d'enfants ayant un retard, les résultats indiquent que les intervenants identifient plus souvent des problèmes d'alcool et de drogues, de santé mentale et physique, de soutien social, d'ordre économique, des activités criminelles et de retard mental. En somme, plusieurs des caractéristiques qui distinguent les enfants qui ont un retard des autres enfants signalés à la DPJ semblent être des indicateurs de la présence de problèmes et de difficultés au sein des familles de ces enfants.

\section{Caractéristiques des décisions rendues par la DPJ}

Nos résultats indiquent notamment que les signalements d'enfants qui ont un retard sont plus souvent jugés fondés, et dans ces cas, la sécurité et le développement sont plus souvent considérés comme étant compromis, ce qui est similaire aux résultats de Fuchs et ses collègues (2007). étant donné les besoins particuliers que ces jeunes nécessitent, le retard de l'enfant peut être considéré comme un indicateur important que la sécurité et le développement soient compromis, et en ce sens, les intervenants prendraient en considération cet aspect.

De même, comparativement aux autres enfants, les résultats de notre étude démontrent que ceux présentant un retard ont plus souvent été pris en charge en CJ dans les cinq dernières années. Ces résultats correspondent à ceux trouvés dans la littérature (Connell et al., 2007 ; Marshall \& English, 1999), ceux-ci stipulant que les enfants qui présentent un retard sont plus à risque de se voir référer à nouveau à la protection de l'enfance. Le fait d'avoir déjà été pris en charge au cours des cinq dernières années indique un problème chronique au sein de la famille et pourrait supposer que les interventions apportées par les centres jeunesse n'ont pas su répondre suffisamment ou adéquatement aux problèmes vécus dans ses familles.

En ce qui a trait aux cas d'enfants dont la sécurité et le développement sont compromis, nos résultats indiquent que la prise en charge est plus fréquemment volontaire ou judiciarisée. Effectivement, il est plus rare que survienne une fermeture du dossier via une intervention terminale par exemple. Les intervenants estiment donc que la prise en charge par la DPJ des enfants ayant un retard est nécessaire afin que la sécurité et le développement ne soient plus compromis. D'ailleurs, les intervenants identifient dans presque la totalité des cas que les enfants ayant un retard ont besoin de services. Ce résultat est particulièrement intéressant, car plusieurs auteurs mentionnent plutôt que les intervenants ont de la difficulté à identifier les besoins de ces enfants (ex., Shannon, 2006). Bien que les intervenants soient en mesure d'identifier les 
services et interventions nécessaires au bon développement et à la sécurité de ces jeunes, il est possible que ceux-ci ne les reçoivent pas (Fuchs et al., 2007 ; Krahn et al. 2000 ; Orelove et al., 2000), ce que cette étude n’a pu évaluer. Néanmoins, le fait que ces enfants aient déjà été pris en charge par le passé peut indiquer qu'ils n'ont pas reçu les services requis ou, s'ils ont été reçus, qu'ils n’ont pas été efficaces ou adéquats.

Contrairement aux résultats de plusieurs études (Fuchs et al., 2007 ; Jones, 1993 ; Romney et al., 2006), les enfants qui présentent un retard n'ont pas fait l'objet d'une recommandation de placement dans une plus grande proportion. Ainsi, il est probable que d'autres facteurs aient plus de poids sur la décision de placer ou non un enfant (voir Tourigny, Poirier, Dion, \& Boisvert, sous presse, pour une étude sur les facteurs liés au placement). Des études futures sont toutefois nécessaires afin d'éclairer ces résultats.

\section{Forces et limites de l'étude}

La présente étude a permis d'accroître nos connaissances sur les caractéristiques individuelles, familiales et liées aux services de protection des enfants qui ont des retards. Parmi les forces de l'étude, notons l'utilisation d'un échantillon représentatif des signalements québécois, provenant des 18 régions sociosanitaires du Québec durant une période de trois mois, un nombre de sujets élevé permettant des analyses multivariées sur un grand nombre de variables recueillies par les intervenants des CJ et l'utilisation d'un devis de recherche prospectif, les données étant recueillies à la suite du signalement. Quant aux limites de l'étude, il est à souligner que certains facteurs pertinents n'ont pas pu être retenus dans les analyses (p. ex., secondaire complété ou non, niveau socio-économique), en raison d'un trop grand nombre de données manquantes pour ces variables. Par ailleurs, les enfants qui ont un retard intellectuel, développemental et langagier ont été regroupés afin d'obtenir un nombre plus élevé d'enfants qui présentent un retard. Bien que ces enfants soient les plus à risques d'être victimes de maltraitance (Sullivan \& Knutson, 2000), ils peuvent présenter des caractéristiques particulières qui diffèrent d'un type de retard à l'autre.

\section{Implications cliniques}

Les résultats de cette étude démontrent que les enfants ayant un retard présentent des caractéristiques différentes de ceux n'en présentant pas, et ce, à différentes étapes du processus d'évaluation et d'orientation des signalements à la protection de la jeunesse. De façon importante, les intervenants identifient plus souvent que ces enfants ont besoin de services. Ces résultats suggèrent ainsi des besoins distincts lorsque vient le temps de développer un plan d'intervention pour ces enfants. Afin de mieux identifier ces besoins, il importe qu'une évaluation complète et spécialisée des enfants qui ont un retard soit effectuée. Plus particulièrement, comme ces enfants sont surreprésentés dans les cas de négligence et proviennent en général de familles plus pauvres et de 
milieux de résidence moins sécuritaire, il faudrait alors envisager des modalités particulières dans la prise en charge de ces familles. Par exemple, des services de soutien devraient être offerts aux familles d'enfants qui ont des retards (Fudge Schormans \& Brown, 2005 ; Sullivan \& Knuston, 2000 ; Sobsey, 1994), notamment pour accroître, auprès des parents, les connaissances et leur compréhension du développement de leur enfant, renforcer leurs habiletés parentales, les sensibiliser aux ressources existantes, diminuer l'isolement vécu et les aider à développer des stratégies d'adaptation positives (Department of Health \& Human Services USA, 2001 ; Senn, 1988).

Cette étude a également permis de faire ressortir que les enfants ayant un retard présentent des caractéristiques liées aux services dont celles liées à certaines décisions qui diffèrent des autres enfants. Bien que notre étude ne permette pas de statuer sur le bien-fondé ou non des décisions prises dans les cas de signalements impliquant des enfants ayant des retards, ces enfants semblent toutefois bénéficier d'interventions plus rapides, plus longues, plus souvent fondées, suggérant que les intervenants sociaux semblent reconnaitre les besoins particuliers de ces enfants, à tout le moins lors du processus d'évaluation et d'orientation. Toutefois et d'une manière plus globale, la littérature actuelle montre qu'il faut offrir aux intervenants des outils adaptés et valides et des formations spécialisées pour intervenir auprès de ces enfants (Hughes \& Rycus, 1998 ; Lightfoot \& Laliberte, 2006 ; Shanon \& Agorastou, 2006 ; Wescott \& Jones, 1999). De plus, il faut être en mesure de mieux identifier les enfants qui ont un retard, et ce, à l'aide de meilleurs instruments d'évaluations (Hughes \& Rycus, 1998 ; Shanon \& Agorastou, 2006). Accroître l'accès à des traitements appropriés est un autre défi auquel les intervenants doivent s'adresser en priorité (Shannon, 2006). Finalement, un suivi plus serré de ces enfants s'impose. S'assurer qu'ils reçoivent les services qui ont été identifiés par les intervenants, mettre en place des mesures pour garantir la sécurité des enfants sont quelques exemples du suivi requis pour éviter la reprise en charge par la DPJ.

\section{CONCLUSION}

Les résultats de cette étude offrent un premier aperçu des caractéristiques des enfants qui présentent un retard et qui sont signalés à la DPJ. De façon générale, plusieurs caractéristiques individuelles et familiales différencient les enfants qui ont un retard des autres enfants. Par exemple la négligence, l'aide sociale (une variable proxy de la pauvreté), et le fait d'avoir été pris en charge dans les cinq dernières années semblent être des indicateurs présents au sein des familles qui pourraient expliquer ou être des effets du retard chez l'enfant, étant donné le caractère bilatéral des effets de la maltraitance. De plus, le retard de l'enfant semble être pris en considération par les intervenants dans leurs décisions. Toutefois, comme la prise en charge dans les cinq dernières années distingue les deux groupes, il est possible de supposer qu'il y a encore des problèmes dans les familles des enfants qui ont des retards, malgré les interventions passées. De 
futures recherches sont donc nécessaires afin de déterminer comment les services peuvent mieux répondre aux besoins de cette population (Weaver, 2005). Dans un contexte où il y a augmentation du nombre d'enfants à besoins spéciaux dans les centres de protection de l'enfance (Marchenski \& Mudry, 2005), le besoin de professionnels compétents pouvant les soutenir et les protéger adéquatement est criant (Weaver, 2005).

\section{Remerciements}

Les auteurs souhaitent remercier les intervenants de la Direction de la protection de la jeunesse du Québec pour leur précieuse collaboration à ce projet ainsi que Pierre McDuff pour ses judicieux commentaires. Cette étude a été réalisée dans le cadre des recherches du Centre de recherche interdisciplinaire sur les problèmes conjugaux et les agressions sexuelles (CRIPCAS). Elle a bénéficié d'une subvention du Programme d'aide institutionnelle à la recherche de l’Université du Québec à Chicoutimi.

\section{RÉFÉRENCES}

Buchele-Ash, A., Turnbull, H. R., \& Mitchell, L. (1995). Forensic and law enforcement issues in the abuse and neglect of children with disabilities. Mental \& Physical Disability Law Reporter, 19, 115-121.

Coicchinelli, L. F. (1991). Proceedings of the symposium on risk assessment in child protective services. Washington, DC: National Center on Abuse and Neglect.

Comité d'experts sur la révision de la Loi sur la protection de la jeunesse (2004). La protection des enfants au Québec: Une responsabilité à mieux partager. Québec : Direction générale des services à la population, ministère de la Santé et des Services sociaux.

Cooke, P., \& Standen, P. J. (2002). Abuse and disabled children: Hidden needs. Child Abuse Review, 11, 1-18.

Connell, C. M., Bergeron, N., Katz, K. H., Saunders, L., \& Tebes, J. K. (2007). Re-referral to child protective services: The influence of child, family, and case characteristics on risk status. Child Abuse \& Neglect, 31, 573-588.

Departement of health \& human services USA. (2001). The risk and prevention of maltreatment of children with disabilities. Child Welfare Information Gateway.

Fuchs, D., Burnside, L., Marchenski, S., \& Mudry, A. (2007). Children with disabilities involved with the child welfare system in Manitoba: Current and future challenges. Dans I. Brown, F. Chaze, D. Fuchs, J. Lafrance, S. McKay, \& S. Thomas Prokop (Eds.), Putting a buman face on child welfare: Voices from the Prairie. Prairie Child Welfare Consortium www.uregina.ca/spr/prairechild/index.html/Centre of Excellence for Child Welfare www.cecw-cepb.ca

Fudge Schormans, A., \& Brown, I. (2005). An investigation into the characteristics of the maltreatment of children with developmental delays and the alleged perpetrators of this maltreatment. Journal on Developmental Disabilities, 9, 1-19. 
Fudge Schormans, A., \& Sobsey, D. (2007). Maltreatment and developmental Disability. Dans I. Brown \& M. Percy (Eds.), Intellectual and developmental disabilities: International perspectives. Baltimore: Paul H. Brookes.

Goldson, E. (1998). Children with disabilities and child maltreatment. Child Abuse \& Neglect, 22, 663-667.

Hershkowitz, I., Horowitz, D., \& Lamb, M. E. (2007). Victimization of children with disabilities. American Journal of Orthopsychiatry, 4, 629-635.

Hosmer, D. W., \& Lemeshow, S. (1980). Goodness of fit test for the multiple logistic regression model. Communications in Statistic, Theory and Method, A9, 1043-1069.

Hughes, R. C., \& Rycus, J. S. (1998). Developmental disabilities and child welfare. Washington, DC: CWLA Press.

Jacob, M., \& Laberge, D. (2001). L'évaluation des signalements à la Direction de la protection de la jeunesse : étude des facteurs qui influencent les décisions prises par les intervenants. Criminologie, 34, 123-151.

Jones, J. (1993). Decision making in child welfare: A critical review of the literature. Child and Adolescent Social Work Journal, 10, 241-259.

Krahn, G. L., Thom, V. A., Sokoloff, K., Hylton, J., \& Steinberg, M. (2000). Every child special, every child safe: Protecting children with disabilities from maltreatment. Portland, OR: Oregon Health Sciences University.

Kvam, M. H. (2000). Is sexual abuse of children with disabilities disclosed? A retrospective analysis of child disability and the likelihood of sexual abuse among those attending Norwegian hospitals. Child Abuse \& Neglect, 24, 1073-1084.

Lightfoot, E. B., \& LaLiberte, T. L. (2006). Approaches to child protection case management for cases involving people with disabilities. Child Abuse \& Neglect, 30, 381-391.

Manders, J. E, \& Stoneman, Z. (2009). Children with disabilities in the child protective services system: An analog study of investigation and case management. Child abuse \&o neglect, 33, 229-37.

Marchenski, S., \& Mudry, A. (2005). Children with disabilities involved with the child and family services system: Understanding the context. The Manitoba Journal of Child Welfare, 4, 1-15.

Marshall, D. B., \& English, D. J. (1999). Survival analysis of risk factors for recidivism. Child Abuse \& Neglect, 4, 287-296.

Orelove, F. P., Hollahan, D. J., \& Myles, K. T. (2000). Maltreatment of children with disabilities: Training needs or a collaborative response. Child Abuse \& Neglect, 24, 185-194.

Perona, A. R., Bottoms, B. L., \& Sorenson, E. (2006). Research-based guidelines for child forensic interviews. Journal of Aggression, Maltreatment, \& Trauma, 12, 81-130.

Reinke, D. C. (2005). Similarities and differences between children with and without disabilities on identified clinical findings. Developmental Disabilities Bulletin. Special Issue: Abuse and Disabilities, 33, 44-70.

Romney, S. C., Litrownik, A. J., Newton, R. R., \& Lau, A. (2006). The relationship between child disability and living arrangement in child welfare. Child Welfare, 85 , 965-984.

Senn, C. Y. (1988). Vulnerable: Sexual abuse and people with an intellectual handicap. Toronto: G. Allan Roeher Institute. 
Sedlak, A. (1991). National incidence and prevalence of child abuse and neglect: 1988. Rockville, MD : Westat.

Shannon, P., \& Agorastou, M. (2006). Identifying children with developmental disabilities receiving child protection services: A national survey of child welfare administrators. Families in Society, 87, 351-357.

Shannon, P. (2006). Children with disabilities in child welfare. In Boyd Webb, N. (Ed.). Working with Traumatized Youth in Child Welfare (pp. 155-170). New York: Guilford Press.

Sobsey, D. (1994). Violence and abuse in the lives of people with disabilities. The end of silent acceptance? Baltimore: Brookes.

Sobsey, D., \& Mansell, S. (1994). Sexual abuse patterns of children with disabilities. The International Journal of Children's Rights, 2, 96-100.

Sobsey, D., Randall, W., \& Parrila, R. K. (1997). Gender differences in abused children with and without disabilities. Child Abuse \& Neglect, 21, 707-720.

Strickler, H. L. (2001). Interaction between family violence and mental retardation. Mental Retardation, 6, 461-471.

Sullivan, P. M., \& Knutson, J. F. (2000). Maltreatment and disabilities: A populationbased epidemiological study. Child Abuse \& Neglect, 24, 1257-1273.

Sullivan, P. M., \& Knutson, J. F. (1998). The association between child maltreatment and disabilities in a hospital-based epidemiologica study. Child Abuse \& Neglect, 22, 271-288.

Tabachnick, B. G., \& Fidell, L. S. (2007). Using multivariate statistics, 5th ed. Boston: Allyn and Bacon.

Tharinger, D., Horton, C. B., \& Millea, S. (1990). Sexual abuse and exploitation of children and adults with mental retardation and others handicaps. Child Abuse \& Neglect, 14, 301-312.

Tourigny, M., Mayer, M., Wright, J., Lavergne, C., Trocmé, N., Hélie, S., Bouchard, C., Chamberland, C., Cloutier, R., Jacob, M., Boucher, J. \& Larrivée, M.-C. (2002). étude sur l'incidence et les caractéristiques des situations d'abus, de négligence, d'abandon et de troubles de comportement sérieux signalées à la direction de la protection de la jeunesse au Québec (ÉIQ). Montréal : Centre de liaison sur l'intervention et la prévention psychosociales.

Tourigny, M., Poirier, M-A, Dion, J., \& Boisvert, I. (sous presse). Recommandation de placement de l'enfant dans le contexte de la protection de la jeunesse : Facteurs associés. Revue de psychoéducation.

Trocmé, N., McPhee, D., Tam, K. K., \& Hay, T. (1994). Ontario incidence study of reported child abuse and neglect. Toronto: Institute for the Prevention of Child Abuse.

Trocmé, N., MacLaurin, B., Fallon, B., Daciuk, J., Billingsley, D., Tourigny, M., Mayer, M., Wright, J., Barter, K., Burford, G., Hornick, J., Sullivan, R., \& McKenzie, B. (2001). étude canadienne sur l'incidence des signalements de cas de violence et de négligence envers les enfants : Rapport final. Ottawa : ministère de Travaux publics et Services gouvernementaux Canada.

Trocmé, N., Fallon, B., MacLaurin, B., Daciuk, J., Felstiner, C., Black, T., Tonmyr, L., Blackstock, C., Barter, K., Turcotte, D., \& Cloutier, R. (2005). étude canadienne sur l'incidence des signalements de cas de violence et de négligence envers les enfants 2003 : Données principales. Ottawa : ministre des Travaux publics et des Services gouvernementaux du Canada. 
Vézina, A., \& Bradet, D. (1990). Inventaire concernant le bien-être de l'enfant en relation avec l'exercice des responsabilités parentales. Québec : MSSS et Centre de recherche sur les services communautaires.

Weaver, C. J. (2005). Children with disabilities in the child welfare system. Récupéré le 16 mai 2009 de http://www.socialworktoday.com/archive/swt_0505p24.htm

Westcott, H. L., \& Jones, D. P. H. (1999). Annotation: The abuse of disabled Children. Journal of Child Psychology and Psychiatry, 40, 497-506. 